\title{
Consumo de MDMA (éxtasis) en estudiantes de secundaria
}

\author{
Sálz Martínez, P.A.; González García-Portilla, M.P.; Paredes Ojanguren, B.; Delgado González, J.M.; \\ López Rodríguez, J.L.; Martínez Barrondo, S.; Bobes García, J.
}

Area de Psiquiatría - Facultad de Medicina (Universidad de Oviedo)

Enviar correspondencia a:

Pilar Alejandra Sáiz Martínez. Area de Psiquiatría - Facultad de Medicina. Julián Clavería 6 - 3º. 33006 Oviedo. Tfno / FAX: 985103552. E-mail: frank@correo.uniovi.es

\section{RESUMEN}

Objetivos: Describir la prevalencia de consumo de MDMA y el perfil toxicológico y psicológico en una muestra de estudiantes de secundaria.

Sujetos y Método: Durante el curso académico 1998-99 se evaluó una muestra compuesta por 2.862 jóvenes de ambos sexos [edad media (SD): 15.87 (1.48); 50.6\% hombres], estudiantes de secundaria de Oviedo (Asturias).

Evaluación: Cuestionario de la OMS de consumo de alcohol y drogas, cuestionario de personalidad de Eysenck versión adultos (EPQ-A) y, la escala de búsqueda de sensaciones de Zuckerman (EBS).

Resultados: Las prevalencias vida, último año y último mes, de consumo de MDMA fueron 3.8\%, 2.7\% y $1.6 \%$, ocupando la MDMA la $7^{a}$ posición entre las drogas ilegales consumidas alguna vez. Los hombres mostraron mayores prevalencias de consumo, vida, último año y último mes, que las mujeres $(p<$ .05). Comparados con los estudiantes que nunca han consumido MDMA (pero que pueden haber consumido otras drogas ilegales), los usuarios de MDMA refieren una historia más extensa de uso de drogas. La MDMA es, tras la heroína, la droga asociada a un mayor índice de policonsumo. Los jóvenes que han usado MDMA alguna vez obtuvieron puntuaciones más elevadas en la subescala de psicoticismo del EPQ-A y refirieron niveles más elevados de búsqueda de sensaciones.

Conclusiones: Los consumidores de MDMA son policonsumidores de otras sustancias legales e ilegales. Aquellos que consumen MDMA tienen un perfil psicológico diferente, caracterizado por elevados niveles de búsqueda de sensaciones y psicoticismo.

Palabras Clave: MDMA, Estudiantes de Secundaria, Epidemiología, Personalidad, Búsqueda de Sensaciones, Policonsumo, Sexo

\section{SUMMARY}

Objectives: To describe the prevalence of MDMA consumption and the toxicological and psychological profile in a sample of secondary school students

Subjects and Method: During the 1998-99 school year, a sample of 2,862 young people from Oviedo (Asturias) of both sexes [mean age (SD): $15.87(1.48)$ ]; 50.6\% males] were studied.

Evaluation: WHO Drug Consumption Questionnaire, Eysenck Personality Questionnaire, Adult Version (EPQ-A), and Zuckerman Sensation Seeking Scale (SSS).

Results: The prevalence of lifetime, previous year and previous month MDMA use was $3.8 \%, 2.7 \%$ and $1.6 \%$, ranking it 7 th among illicit drugs ever used. Males showed a higher lifetime, previous year, and previous month consumption of MDMA than females $(p<.05)$. Compared to students who had never used MDMA (but who may have used other illicit drugs) MDMA users had a more extensive drug abuse history. MDMA is, after heroin, the drug with the highest polyconsumption level. Lifetime MDMA consumers had significantly higher scores on the EPQ-A psychoticism subscale of the EPQ-A, and showed higher levels of sensation-seeking.

Conclusions: MDMA consumers are polyconsumers of other legal and illegal substances. Those who consume MDMA have a different psychological profile, characterized by high sensation-seeking and high levels of psychoticism.

Key Words: MDMA, Secondary School Students, Epidemiology, Personality, Sensation Seeking, Polyconsumption, Gender

\section{INTRODUCCION}

B ajo el epígrafe de drogas de síntesis se agrupan una serie de sustancias psicoactivas creadas a partir de la modificación de la estructura quími- ca de determinados productos naturales o de determinadas sustancias utilizadas como medicamentos, que en su mayor parte adoptan la forma de "pastillas", y que pueden ser fácilmente sintetizadas por métodos químicos sencillos. 
La más conocida de todas ellas es la MDMA (3,4metilendioximetanfetamina), sustancia perteneciente al grupo de las feniletilaminas (de estructura parecida a la anfetamina, pero dotada de un anillo bencénico y un grupo amino), más conocida vulgarmente como éxtasis. Esta sustancia, sintetizada por los laboratorios E. Merck en 1912, no comienza a ser utilizada tanto a nivel terapeútico como recreacional, en Estados Unidos, hasta la década de los setenta (Greer y Tolbert, 1986; Grinspoon y Bakalar, 1986; Bobes et al, 1998a).

El interés por la MDMA comienza a dispararse cuando en 1978, Shulgin y Nichols (1978) sugieren la posibilidad de su utilización como coadyuvante terapeútico. En el año 1985 la Drug Enforcement Agency americana incluye, por procedimiento urgente, la MDMA en la Lista I de la Comprehensive Substances Act, quedando prohibida su producción, venta, posesión y consumo con fines terapeútico o recreacional. Dicha inclusión se acompañó de un gran revuelo en los medios de comunicación estadounidenses, cuyo resultado fue un incremento de la popularidad de la sustancia (Beck, 1986). En Europa, la droga comienza a ser conocida a mediados de los ochenta, siendo inicialmente más popular en países como el Reino Unido, Holanda, Alemania y España, donde es utilizada por grupos reducidos ("fashionable elite") de jóvenes (Griffiths et al, 1997).

Los datos actuales sugieren que el consumo de MDMA existe en prácticamente todos los países europeos (si bien, en algunos, como los países Nórdicos o Grecia, es un fenómeno relativamente reciente), generalmente asociado a determinados ambientes lúdicos en los que unos tipos de música y el baile concretos (techno/house) ocupan un lugar estelar, siendo, en dichos ambientes, frecuente el consumo de psicoestimulantes (anfetaminas / metanfetaminas y sus derivados y cocaína), alucinógenos (LSD) y otras sustancias como cannabis y alcohol. En general, los usuarios clásicos de esta sustancia no suelen consumir opiáceos, pero existen, no obstante, referencias de consumo de heroína entre policonsumidores.

España es uno de los países europeos con tasas más elevadas de consumo de esta sustancia (DGPNSD, 1996a; Gamella y Alvarez, 1997; Griffiths et al, 1997; Bobes et al, 1998b; Calafat et al, 1998; Sáiz et al, 1998). Concretamente, a partir del año 1992 se ha producido, en nuestro país, una masificación de su consumo, que ha pasado de ser un producto ideologizado (con contenidos espirituales, terapeúticos o contraculturales) a ser de consumo masivo, siendo utilizada por decenas de miles de usuarios (Gamella et al, 1996; Alvarez y Gamella, 1998; Bobes et al, 1998b; Calafat et al, 1998).

En el momento actual, se sabe que la MDMA tiene efectos neurotóxicos sobre los terminales serotonérgicos del sistema nervioso central (Ricaurte et al, 1990; McCann et al, 1998), si bien las consecuencias funcionales de dicho daño aún no son bien conocidas (Grob et al, 1998; McCann y Ricaurte, 1998). De igual modo, en los últimos diez años se han realizado numerosas publicaciones en las que se ponen de manifiesto, tanto diversos efectos adversos de tipo físico y psíquico, como algunos casos de muerte en los que estaba implicado el consumo de esta sustancia (Bobes et al, 1995, 1998c; Gamella y Alvarez, 1997; Griffiths et al, 1997).

Los objetivos de este estudio son los siguientes: 1) determinar la prevalencia de consumo de MDMA en estudiantes de secundaria de ambos sexos 2) establecer el perfil toxicológico y psicológico de los consumidores de MDMA.

\section{MATERIAL Y METODOS}

Durante el curso académico 1998-1999, se ha estudiado una muestra compuesta por 2.915 jóvenes, de ambos sexos, alumnos de enseñanza secundaria (cursos $2^{\circ}$ ESO, $3^{\circ}$ ESO, $4^{\circ}$ ESO y $1^{\circ}$ Bachiller) de los centros públicos de Oviedo (Asturias).

Dichos jóvenes constituyen el total de los alumnos que cursan estudios en centros públicos de Oviedo y que estaban en sus respectivas clases en el momento de la cumplimentación del protocolo. Todos ellos cumplimentaron personalmente y bajo la supervisión de uno de los miembros del equipo investigador el siguiente protocolo: cuestionario de la OMS de consumo de alcohol y drogas (Smart et al, 1980), el cuestionario de personalidad de Eysenck en su versión para adultos (EPQ-A) (Eysenck y Eysenck, 1975) y la escala de búsqueda de sensaciones de Zuckerman en su forma $V$ (EBS) (Zuckerman et al, 1978).

En el análisis estadístico de las variables cuantitativas se utilizó el test t de Student para muestras independientes o un análisis de la varianza (Anova Oneway), utilizando el test de Duncan para clarificar la naturaleza de los hallazgos realizados tras la realización del anova. Para las variables cualitativas se utilizó la prueba chi cuadrado con corrección de Yates y el test exacto de Fisher. En todos los casos el nivel de significación estadística quedó establecido en el 5\%.

\section{RESULTADOS}

Para el análisis de resultados se contó con un total de 2.862 protocolos válidos $(98,2 \%$ de los 2.915 protocolos iniciales). Fueron excluidos un total de 53 protocolos (21 de ellos por no alcanzar el percentil 15 en la escala de sinceridad del EPQ-A, y 32 protocolos por estar incompletos). De los protocolos válidos, 1.448 
(50.6\%) correspondían a hombres, mientras que el resto pertenecían a mujeres (1.414 protocolos).

\section{Análisis general de la muestra}

Como ya se ha comentado con anterioridad la muestra estaba constituída por 2.862 jóvenes de ambos sexos de edades comprendidas entre 13 y 30 años [edad media (SD): 15.87 (1.48)]. En la tabla 1 se muestran las prevalencias vida, último año y último mes del consumo de drogas de la muestra global, así como la edad de inicio de consumo de las diferentes sustancias evaluadas. Como puede verse en dicha tabla, las sustancias más comúnmente consumidas por estos jóvenes son el alcohol y el tábaco (con prevalencias vida de $90.3 \%$ y $66.5 \%$, respectivamente) seguidas a cierta distancia por el cannabis (prevalencia vida del $35.2 \%$ ). La prevalencia vida del consumo del resto de sustancias es obstensiblemente más baja, no alcanzando en ningún momento cotas superiores al $11 \%$. Como era de esperar las prevalencias de consumo disminuyen a medida que acotamos el período explorado, si bien el ranking de preferencias tiende a mantenerse estable (ver prevalencias de consumo en el último año y último mes en la tabla 1). De igual modo, puede observarse que la MDMA ocupa la $9^{a}$ posición en el ranking de preferencias, tanto alguna vez en la vida, como en el último año y último mes.
En lo que a edad de inicio se refiere se constata que las primeras sustancias que comienzan a utilizar estos jóvenes son las drogas legales, tabaco y alcohol, con una edad media de inicio (SD) de 13.12 (2.09) y 13.25 (2.18) respectivamente, seguidas de inhalantes, opiáceos, tranquilizantes y cannabis. La incorporación al consumo de MDMA es un poco más tardía, con una edad media de 15.54 (2.02) años, sólo superada por la edad media de inicio de consumo de cocaína.

Un análisis más detallado del patrón de uso de MDMA revela que, de los 47 jóvenes que han consumido MDMA durante el último mes, 27 (57.45\%) lo han utilizado de 1 a 5 días, 12 (25.53\%) entre 6 y 19 días y, finalmente, $8(17.02 \%)$ más de 19 días. El porcentaje de jóvenes que consumen MDMA como única droga ilegal es muy bajo, así, todos los que han consumido MDMA alguna vez en la vida, han probado alguna vez otras drogas ilegales y tan sólo 5 de los consumidores-vida de MDMA no han tomado otras drogas ilegales durante el último año (lo cual representa un $4.6 \%$ de los consumidores-vida de MDMA).

En la tabla 2 se muestran los índices de policonsumo de cada droga, es decir, el número medio de sustancias consumidas por los consumidores de cada una de las diferentes sustancias. Como puede apreciarse en dicha tabla las tasas medias de policonsumo más elevadas se dan entre los consumidores de heroína, seguido de los consumidores de psicoestimulantes (MDMA, cocaína, anfetaminas) y alucinógenos.

TABLA I: Prevalencia de consumo de drogas y edad de inicio al mismo de la muestra global

\begin{tabular}{|l|c|c|c|c|}
\hline Droga & Alguna vez & Ultimos 12 meses & Ultimo mes & Edad inicio (SD) \\
\hline Tabaco & $1904(66.5 \%)$ & $788(27.5 \%)^{*}$ & $1261(44.1 \%)^{* * *}$ & $13.12(2.09)$ \\
Alcohol & $258(16.6 \%)^{* *}$ & $2111(73.8 \%)$ & $13.25(2.18)$ \\
Cannabis & $1008(35.2 \%)$ & $2431(84.9 \%)$ & $605(21.1 \%)$ & $14.67(4.23)$ \\
Cocaína & $174(6.1 \%)$ & $139(4.9 \%)$ & $78(2.7 \%)$ & $15.66(2.04)$ \\
Anfetaminas & $238(8.3 \%)$ & $163(5.7 \%)$ & $106(3.7 \%)$ & $15.11(1.93)$ \\
Alucinógenos & $309(10.8 \%)$ & $238(8.3 \%)$ & $141(4.9 \%)$ & $15.16(1.72)$ \\
Inhalantes & $230(8.0 \%)$ & $141(4.9 \%)$ & $82(2.9 \%)$ & $13.75(2.93)$ \\
Tranquilizantes & $305(10.7 \%)$ & $207(7.2 \%)$ & $111(3.9 \%)$ & $14.47(2.14)$ \\
Sedantes & $56(2.0 \%)$ & $44(1.5 \%)$ & $24(0.8 \%)$ & $14.71(2.87)$ \\
Opio (fumado) & $74(2.6 \%)$ & $45(1.6 \%)$ & $26(0.9 \%)$ & $14.95(1.79)$ \\
Heroína & $47(1.6 \%)$ & $32(1.1 \%)$ & $25(0.9 \%)$ & $14.85(2.04)$ \\
Otros opiáceos & $50(1.7 \%)$ & $32(1.1 \%)$ & $21(0.7 \%)$ & $14.45(2.40)$ \\
Extasis & $109(3.8 \%)$ & $76(2.7 \%)$ & $47(1.6 \%)$ & $15.54(2.02)$ \\
Otras drogas & & $106(3.7 \%)$ & & \\
\hline
\end{tabular}

*Alguna vez fumó a diario durante 6 meses o más

**Alguna vez fumó sólo los fines de semana durante 6 meses o más

***Actualmente fuma 
TABLA II: Indices de policonsumo de las diferentes drogas según sexo

\begin{tabular}{|l|c|c|c|c|}
\hline Droga & Total $(\mathrm{n}=2.862)$ & Hombres $(\mathrm{n}=1.448)$ & Mujeres $(\mathrm{n}=1.414)$ & $\mathrm{p}^{*}$ \\
\hline Tabaco & $2.27(1.81)$ & $2.55(2.07)$ & $2.05(1.54)$ & .000 \\
Alcohol & $1.71(1.83)$ & $1.80(2.05)$ & $1.64(1.59)$ & .033 \\
Cannabis & $3.28(1.92)$ & $3.53(2.13)$ & $3.04(1.66)$ & .000 \\
Cocaína & $6.01(2.25)$ & $6.01(2.40)$ & $6.00(1.88)$ & $\mathrm{NS}$ \\
Anfetaminas & $5.76(2.15)$ & $5.85(2.33)$ & $5.63(1.83)$ & $\mathrm{NS}$ \\
Alucinógenos & $5.28(2.19)$ & $5.29(2.34)$ & $5.29(1.89)$ & $\mathrm{NS}$ \\
Inhalantes & $4.80(2.64)$ & $4.83(2.89)$ & $4.81(2.18)$ & $\mathrm{NS}$ \\
Tranquilizantes & $4.09(2.56)$ & $4.81(3.06)$ & $5.61(1.99)$ & .000 \\
Sedantes & $6.46(3.27)$ & $7.33(3.79)$ & $7.46(2.21)$ & .026 \\
Heroína & $7.74(2.69)$ & $7.86(2.93)$ & $6.19(1.97)$ & $\mathrm{NS}$ \\
MDMA & $6.48(2.45)$ & $6.61(2.66)$ & $\mathrm{NS}$ \\
\hline
\end{tabular}

*Prueba t de Student

\section{Análisis de la muestra en función del sexo}

La muestra estaba compuesta por 1.448 hombres (50.6\%) y 1.414 mujeres (49.6\%), siendo la edad media de ambas muestras similar [15.85 (1.55) para los hombres versus 15.88 (1.41) para las mujeres; $p=$ 614].

En la tabla III se muestran las prevalencias de consumo de las diferentes drogas en función del sexo. Como puede observarse en dicha tabla, en líneas generales, existe un mayor porcentaje de hombres consumidores de drogas ilegales, si bien cabe señalar que en el caso del cannabis sólo se observan diferencias en el porcentaje de consumidores durante el último mes (más elevado en el caso de los hombres, $\mathrm{p}=$ .012), mientras que las mujeres son más consumidoras de tabaco, alcohol y tranquilizantes. En el caso concreto de la MDMA se observa una mayor proporción de hombres consumidores de la sustancia, tanto alguna vez en la vida $(p=.001)$, como en el último año $(p=.005)$ y durante el último mes $(p=.001)$.

La edad media de inicio de consumo de las diferentes drogas es bastante similar en ambos sexos, con la excepción del consumo de tabaco y alcohol (las primeras drogas consumidas por ambos sexos) y de los tranquilizantes, constatándose en los tres casos un comienzo más temprano en los varones [tabaco: 12.85 (2.38) vs 13.35 (1.75), $p=.000$; alcohol: 12.80 (2.54) vs 13.69 (1.64), $p=.000$; tranquilizantes: 14.07 (2.63) vs 14.72 (1.70), $p=.026]$. Los hombres inician el consumo del resto de drogas en un período de edad comprendido entre los 13.58 (3.18) años, momento en que comienzan a consumir inhalantes y los 15.61 (2.26) momento en que empiezan a consumir cocaína, mientras que para las mujeres ese período oscilaría entre los 14.12 (2.20), momento en el que empiezan a consumir inhalantes y los 15.82 (1.17) años, que es cuando comienzan a consumir MDMA.

La edad media de inicio de consumo de MDMA es de 15.39 (2.34) para los hombres y de 15.82 (1.17) para las mujeres $(p=.225)$. Como ya se ha comentado, la MDMA es la última droga incorporada en el repertorio de consumos del sexo femenino, y la penúltima en el casos de los hombres.

En el caso de los hombres las MDMA es la tercera droga que más policonsumos Ileva asociados, por detrás de heroína y tranquilizantes, mientras que en el colectivo de mujeres ocupa la segunda posición en los índices de policonsumo, sólo superada por la heroína. No obstante, cabe señalar que los índices de policonsumos asociados a la MDMA son similares en ambos sexos $(p=.401)$ (ver tabla II).

\section{Consumo de MDMA y perfil psicológico}

Con el fin de facilitar el establecimiento de un posible perfil psicológico (rasgos de personalidad y búsqueda de sensaciones) de los consumidores de MDMA y, dado que es prácticamente imposible localizar consumidores "puros" de MDMA, hemos decido subdividir la muestra en tres subgrupos: grupo 1 (aquellos que sólo han consumido a lo largo de su vida drogas legales -tabaco y / o alcohol-), grupo 2 (aquellos que han consumido alguna vez drogas ilegales entre las que no figura la MDMA) y grupo 3 (aquellos que han consumido alguna vez MDMA, independientemente del resto de consumos legales e ilegales).

Además se analizó si existía alguna diferencia en cuanto a personalidad entre los sujetos que tras haber consumido alguna vez en la vida siguieron consumien- 
TABLA III: Prevalencia de consumo de drogas en función del sexo

\begin{tabular}{|c|c|c|c|c|c|c|c|c|c|}
\hline \multirow[t]{2}{*}{ Droga } & \multicolumn{3}{|c|}{ Alguna vez } & \multicolumn{3}{|c|}{ Últimos 12 meses } & \multicolumn{3}{|c|}{ Último mes } \\
\hline & Hombres & Mujeres & $p^{*}$ & Hombres & Mujeres & $p^{*}$ & Hombres & Mujeres & $p^{*}$ \\
\hline \multirow[t]{2}{*}{ Tabaco } & $865(59.7 \%)$ & $1039(73.4 \%)$ & .000 & $315(21.7 \%) a$ & $473(33.4 \%)$ a & .000 & $555(38.3 \%) c$ & $706(49.9 \%) \mathrm{c}$ & .000 \\
\hline & & & & $204(14.1 \%) b$ & $270(19.1 \%) b$ & .048 & & & \\
\hline Alcohol & $1279(88.3 \%)$ & $1304(92.2 \%)$ & .000 & $1196(82.6 \%)$ & $1235(87.3 \%)$ & NS & $1014(70.0 \%)$ & $1097(77.6 \%)$ & .000 \\
\hline Cannabis & $504(34.8 \%)$ & $505(35.7 \%)$ & NS & $426(29.4 \%)$ & $376(26.6 \%)$ & NS & $334(23.1 \%)$ & $271(19.2 \%)$ & .012 \\
\hline Cocaína & $120(8.3 \%)$ & $54(3.8 \%)$ & .000 & $97(6.7 \%)$ & $42(3.0 \%)$ & .000 & $56(3.9 \%)$ & $22(1.5 \%)$ & .000 \\
\hline Anfetaminas & $145(10.0 \%)$ & $93(6.6 \%)$ & .001 & $99(6.8 \%)$ & $64(4.5 \%)$ & .012 & $64(4.4 \%)$ & $42(3.0 \%)$ & NS \\
\hline Alucinógenos & $201(13.9 \%)$ & $1087.6 \%)$ & .000 & $167(11.5 \%)$ & $71(5.0 \%)$ & .000 & $98(6.8 \%)$ & $43(3.0 \%)$ & .000 \\
\hline Inhalantes & $145(10.0 \%)$ & $85(6.0 \%)$ & .000 & $93(6.4 \%)$ & $48(3.4 \%)$ & .000 & $58(4.0 \%)$ & $24(1.7 \%)$ & .000 \\
\hline Tranquilizantes & $130(8.8 \%)$ & $175(12.4 \%)$ & .004 & $85(5.9 \%)$ & $122(8.6 \%)$ & .005 & $54(3.7 \%)$ & $57(4.0 \%)$ & NS \\
\hline Sedantes & $30(2.1 \%)$ & $26(1.8 \%)$ & NS & $23(1.6 \%)$ & $21(1.5 \%)$ & $N S^{* *}$ & $16(1.1 \%)$ & $8(0.6 \%)$ & $N S^{* *}$ \\
\hline Opio (fumado) & $54(3.7 \%)$ & $20(1.4 \%)$ & .000 & $31(2.1 \%)$ & $14(1.0 \%)$ & $.016^{* *}$ & $21(1.4 \%)$ & $5(0.3 \%)$ & $.002^{* *}$ \\
\hline Heroína & $36(2.5 \%)$ & $11(0.8 \%)$ & $.000 * *$ & $25(1.7 \%)$ & $7(0.5 \%)$ & $.002 * *$ & $20(1.4 \%)$ & $5(0.3 \%)$ & $.004^{* *}$ \\
\hline Otros opiáceos & $34(2.3 \%)$ & $16(1.1 \%)$ & $.015^{* *}$ & $23(1.6 \%)$ & $9(6.4 \%)$ & $.020 * *$ & $17(1.2 \%)$ & $4(0.3 \%)$ & $.007^{* *}$ \\
\hline MDMA & $73(5.0 \%)$ & $36(2.5 \%)$ & .001 & $51(3.5 \%)$ & $25(1.8 \%)$ & .005 & $35(2.4 \%)$ & $12(0.9 \%)$ & $.001 * *$ \\
\hline
\end{tabular}

* Chi cuadrado con corrección de Yates

**Test exacto de Fisher

a. Alguna vez fumó a diario durante 6 meses o más

b. Alguna vez fumó sólo los fines de semana durante 6 meses o más

c. Actualmente fuma

do (presentaban consumo también en el último mes) y los que ya no consumían (no había consumos en el último mes). Para ello creamos una nueva variable, suma de consumo alguna vez en la vida y consumo en el último mes, en la que los sujetos se clasificaban en tres grupos: "abstinentes" (nunca consumieron MDMA, ni alguna vez, ni en el último mes); "experimentadores" (consumieron MDMA alguna vez, pero no en el último mes); y "reincidentes" (consumieron MDMA alguna vez y en el último mes). Por último, cabe señalar que estos análisis se realizarán de forma individualizada para cada sexo.

A la hora de interpretar los datos que se expondrán a continuación es necesario tener en cuenta la presencia de una variable que puede causar confusión: el consumo de otras sustancias. Así, cuando se analizan los consumos alguna vez en la vida, se observa que en ambos sexos se incrementa de modo significativo el número medio de sustancias consumidas al pasar del grupo 1 al grupo 3 [hombres.- grupo 1= 1.19 (0.70), grupo $2=3.84$ (1.49), grupo $3=7.68(2.61), p=.000$; mujeres.- grupo $1=1.45(0.68)$, grupo $2=3.66(1.28)$, grupo $3=7.42(2.02), p=.000]$. Idéntica situación se observa cuando se realizan comparaciones entre abstinentes, experimentadores y reincidentes [hombres.abstinentes $=2.22$ (1.68), experimentadores $=7.17$ (2.23), reincidentes $=8.06$ (3.06), $p=.000$; mujeres. abstinentes $=2.33(1.45)$, experimentadoras $=7.08$ (1.85), reincidentes $=7.83(2.48), p=.000]$. Por tanto, pudiera ser que las diferencias halladas en el perfil psicológico de los distintos grupos fuesen debidas, al menos en parte, al consumo concomitante de otras sustancias.

\section{Consumo de MDMA alguna vez en la vida y EPQ-A}

Las tablas IV y $\mathrm{V}$ muestran los resultados obtenidos en el análisis de la asociación entre uso-abuso de sustancias alguna vez en la vida y el EPQ-A. Los resultados obtenidos en la dimensión neuroticismo ponen de manifiesto que los consumidores de sustancias ilegales diferentes de MDMA (grupo 2), de ambos sexos, son los que obtienen puntuaciones más elevadas en esta dimensión. No obstante, sólo se obtienen diferencias estadísticamente significativas en la submuestra de mujeres: grupo 1 vs grupo 2 (tabla V).

En la dimensión intro-extroversión puede observarse que los consumidores de MDMA (grupo 3) obtienen puntuaciones más elevadas que los no consumidores de drogas ilegales (grupo 1) (tablas IV y V).

Finalmente, en la dimensión de psicoticismo en ambas submuestras de estudiantes se observa que los tres grupos son diferentes entre sí, de tal manera que se puede hablar de un gradiente de psicoticismo en función del consumo: a más sustancias mayor puntuación en psicoticismo.

Desde un punto de vista clínico, es necesario señalar que tan sólo la dimensión de psicoticismo adquiere trascendencia clínica, ya que es la única en la 
que los consumidores de sustancias ilegales (con independencia de que se incluya o no la MDMA), de ambos sexos, se sitúan en rangos iguales o superiores al percentil 85 .

\section{Consumo de MDMA alguna vez en la vida y Bús- queda de Sensaciones}

La relación entre el uso-abuso de sustancias ilegales alguna vez en la vida y la EBS se reflejan en las tablas IV y V. En conjunto, puede decirse que en ambas submuestras las puntuaciones aumentan significativamente en función del consumo, de modo que son significativamente más bajas en los grupos que no consumen sustancias ilegales y más elevadas en el grupo de jóvenes que consumen MDMA (generalmente, junto a otras drogas ilegales).

Desde el punto de vista clínico, este instrumento se comporta de modo muy similar al anterior, mayor desviación respecto a la norma en función de la importancia del consumo (tabla VI). No obstante, la trascendencia clínica es escasa: únicamente en la escala de deshinbición (los grupos 2 y 3) y en la puntuación total (grupo 3), la muestra de estudiantes mujeres se diferencia de la población de referencia (mujeres estudiantes españolas -Pérez y Torrubia, 1986-) 2 ó más desviaciones estándar.

Persistencia en el consumo: diferencias en EPQ-A entre abstinentes, experimentadores y reincidentes

En las tablas VII y VIII se presentan las puntuaciones obtenidas en el EPQ-A por hombres y mujeres. Como puede verse en las dimensiones neuroticismo y intro-extroversión no se observan diferencias entre abstinentes, experimentadores y reincidentes. Sin embargo, en la dimensión psicoticismo se aprecia, en el caso de los hombres, un claro incremento de la puntuación obtenida en función del nivel de consumo de MDMA (tabla VII) y, en el caso de las mujeres, sólo se observan diferencias entre el hecho de haber consumido (experimentadores y reincidentes) o no (abstinentes) MDMA (tabla VIII).

Nuevamente, las diferencias estadísticas sólo tienen significado clínico en la dimensión de psicoticismo; en ambas muestras se observa que experimentadores y reincidentes se sitúan en percentiles clínicamente significativos (superiores al percentil 85) y, los hombres abstinentes están en el percentil 85.

Persistencia en el consumo: diferencias en EBS entre abstinentes, experimentadores y reincidentes

Los resultados de la asociación entre la búsqueda de sensaciones y el consumo de MDMA se muestran en las tablas VII y VIII. En la muestra de hombres se observa, que en la subescala BEX, las mayores puntuaciones son obtenidas por el grupo de experimentadores, seguido del grupo de reincidentes y, por último, de los abstinentes, mientras que en las subescalas DES, SAB y total sólo se observan diferencias entre abstinentes y consumidores (independientemente de que sean experimentadores o reincidentes). Por otra parte, no se observan diferencias en la subescala BEM (tabla VII). En el caso de las mujeres, el panorama varía ligeramente: diferencias entre abstinentes y consumidores en las subescalas BEX, DES y total, diferencias entre abstinentes y reincidentes en la subescala BEM y no hay diferencias entre los tres subgrupos en la subescala SAB (tabla VIII).

Desde el punto de vista clínico (tabla IX), de nuevo son las subescala DES y total, en el sexo femenino, las que mayores desviaciones presentan respecto a la norma, de modo que las estudiantes experimentadoras y reincidentes superan las 2 desviaciones estándar

Tabla IV: Uso-abuso de MDMA alguna vez en la vida y EPQ-A / EBS (varones)

\begin{tabular}{|l|l|l|l|l|l|}
\hline & Grupo 1 $(\mathrm{n}=839)$ & Grupo 2 $(\mathrm{n}=536)$ & Grupo 3 $(\mathrm{n}=73)$ & $\mathrm{p}^{*}$ & Grupos distintos \\
\hline EPQ-A & & & & & \\
Neuroticismo & $12.29(4.77)$ & $13.04(5.14)$ & $12.81(5.56)$ & .023 & No diferencias \\
Intro-extroversión & $14.53(3.56)$ & $15.54(2.98)$ & $15.49(2.69)$ & .000 & 1 vs 2, 3 \\
Psicoticismo & $4.76(2.91)$ & $6.22(3.37)$ & $8.47(3.88)$ & .000 & 1 vs 2 vs 3 \\
\hline Búsqueda Sensaciones & & & & & 1 vs 3 \\
BEM & $7.49(2.44)$ & $7.78(2.09)$ & $8.19(1.92)$ & .009 & 1 vs 2 vs 3 \\
BEX & $4.54(1.49)$ & $5.97(1.82)$ & $6.89(1.76)$ & .000 & 1 vs 2 vs 3 \\
DES & $6.10(1.98)$ & $7.88(1.69)$ & $8.38(1.49)$ & .000 & 1 vs 2 vs 3 \\
SAP & $5.31(1.94)$ & $5.82(1.95)$ & $6.62(2.22)$ & .000 & 1 vs 2 vs 3 \\
Total & $23.42(5.11)$ & $27.43(4.79)$ & $29.96(4.95)$ & .000 & \\
\hline
\end{tabular}

*Anova Oneway (prueba post hoc: Duncan) 
por encima de la norma (Pérez y Torrubia, 1986), en dichas subescalas.

\section{DISCUSION}

Estimar la prevalencia de uso de cualquier droga es una tarea complicada. En el caso de las drogas de síntesis, la naturaleza rápidamente cambiante de su uso y la carencia de métodos adecuados para su registro plantean importantes dificultades (Griffiths et al, 1997).

Los datos de nuestro estudio sugieren que tras el cannabis, los psicoestimulantes (alucinógenos, anfetaminas, cocaína y MDMA) son las drogas ilegales más utilizadas por nuestra población. No obstante, cuando se considera aisladamente el uso de MDMA éste ocupa una situación secundaria, lo cual está en consonancia con la tendencia a la estabilización del consumo de esta sustancia observada en los últimos años en nuestro país (Bobes et al, 1998b).

Durante los últimos años el uso recreacional de MDMA, por parte de los jóvenes, se ha incrementado de una manera marcada en los países de Europa Occidental y los Estados Unidos, generalmente asociado a ciertos tipos de música y determinada escena social (denominada "bakalao" en nuestro país). Datos específicos de España sugieren que el éxtasis es la droga cuyo consumo ha aumentado más deprisa en la última década. Se estima que entre el 5 y el $10 \%$ de jóvenes de edades comprendidas entre los 18-25 años han utilizado la sustancia. Cuando se pone el punto de mira en la población general las cifras descienden, alrededor de 2.2\% en 1994 (CIS, 1994), 1.6\% en 1995 (DGPNSD, 1996b) y 2.5 en 1997 (DGPNSD, 1998). El grupo de edad dónde los consumos son más importantes son los jóvenes de 19-24 años (5\% de consumidores), seguidos de los adultos jóvenes (2530 años) que representan el 3.5\% de consumidores y de los adolescentes (15-18 años), con un 2.5\% de consumidores (DGPNSD, 1996b).

Estudios realizados en población general en Amsterdam muestran incrementos desde el 1.2\% de consumidores en 1990 (Sandwijk et al, 1991) hasta el $3.4 \%$ en 1994 (Sandwijk et al, 1995). Otros estudios realizados en el mismo entorno ponen de manifiesto que a pesar de que la prevalencia de consumo de MDMA es inferior a la de anfetaminas o LSD, el uso reciente es a menudo más elevado (Griffiths et al, 1997). Datos procedentes del Reino Unido sugieren que en 1996 la prevalencia vida de consumo de MDMA en población general es del 3\%. Esas cifras se elevan al 9\% en grupos de población de 16-29 años. El 2\% de dicho grupo de edad habría utilizado la droga durante el último mes, frente a un $1 \%$ de usuarios de LSD y un $4 \%$ de usuarios de anfetaminas (Health Education Authority, 1997).

En Alemania, en el año 1995, la prevalencia vida de consumo de éxtasis fue del $1.6 \%$ en población general (Rabes y Kraus, 1997). Dicha prevalencia era menor que la descrita para anfetaminas y LSD. Sin embargo, durante el último año el uso de MDMA fue ligeramente superior $(0.9 \%)$ que el de las otras dos sustancias (Rabes y Kraus, 1997). En Suiza, el 3.5\% de jóvenes entre 15-34 años han consumido alguna vez MDMA (SFA/ISPA, 1996; Giroud et al, 1997). En otros países europeos como por ejemplo Finlandia las prevalencias son mucho más bajas $(0.2 \%$ de prevalencia vida en población general durante 1996), siendo muy similar lo que ocurre en otros países nórdicos como Suecia y Dinamarca (Kontula, 1997; Olsson, 1997; National Board of Health, 1996).

Estudios realizados específicamente en población escolar aportan cifras más cercanas a las halladas en nuestro estudio. Así, en España, durante el año 1994,

TABLA V: Uso-abuso de MDMA alguna vez en la vida y EPQ-A / EBS (mujeres)

\begin{tabular}{|c|c|c|c|c|c|}
\hline & Grupo $1(n=826)$ & Grupo $2(n=552)$ & Grupo $3(n=36)$ & $p^{*}$ & Grupos distintos \\
\hline EPQ-A & & & & & \\
\hline Neuroticismo & $15.21(4.89)$ & $16.72(4.76)$ & $15.61(4.98)$ & .000 & 1 vs 2 \\
\hline Intro-extroversión & $14.26(3.60)$ & $15.24(3.32)$ & $15.81(2.86)$ & .000 & 1 vs 3 \\
\hline Psicoticismo & $3.32(2.25)$ & $4.53(2.82)$ & $5.47(3.52)$ & .000 & 1 vs 2 vs 3 \\
\hline Búsqueda Sensaciones & & & & & \\
\hline BEM & $6.94(2.51)$ & $7.35(2.25)$ & $7.78(1.88)$ & .003 & 1 vs 3 \\
\hline BEX & $5.12(1.50)$ & $6.62(1.61)$ & $7.64(1.55)$ & .000 & 1 vs 2 vs 3 \\
\hline DES & $4.98(2.12)$ & $6.86(1.90)$ & $7.55(1.44)$ & .000 & 1 vs 2 vs 3 \\
\hline SAP & $4.99(3.47)$ & $5.69(1.79)$ & $5.97(1.92)$ & .000 & 1 vs 3 \\
\hline Total & $21.97(5.13)$ & $26.51(4.63)$ & $29.00(3.94)$ & .000 & 1 vs 2 vs 3 \\
\hline
\end{tabular}

*Anova Oneway (prueba post hoc: Duncan) 
TABLA VI: Diferencias, expresadas en desviaciones estándar, entre las puntuaciones obtenidas por hombres y mujeres y la población española de referencia en la EBS

\begin{tabular}{|c|c|c|c|}
\hline & Grupo 1 & Grupo 2 & Grupo 3 \\
\hline $\begin{array}{l}\text { Búsqueda emociones (BEM) } \\
\text { - hombres } \\
\text { - mujeres }\end{array}$ & $\begin{array}{l}.27 \\
.35\end{array}$ & $\begin{array}{l}.38 \\
.52\end{array}$ & $\begin{array}{l}.53 \\
.70\end{array}$ \\
\hline $\begin{array}{l}\text { Búsqueda excitación (BEX) } \\
\text { - hombres } \\
\text { - mujeres }\end{array}$ & $\begin{array}{l}-.55 \\
-.15\end{array}$ & $\begin{array}{l}.13 \\
.64\end{array}$ & $\begin{array}{r}.57 \\
1.18\end{array}$ \\
\hline $\begin{array}{l}\text { Desinhibición (DES) } \\
\text { - hombres } \\
\text { - mujeres }\end{array}$ & $\begin{array}{r}.40 \\
1.04\end{array}$ & $\begin{array}{l}1.11 \\
2.03\end{array}$ & $\begin{array}{l}1.31 \\
2.39\end{array}$ \\
\hline $\begin{array}{l}\text { Susceptibilidad aburrimiento (SAB) } \\
\text { - hombres } \\
\text { - mujeres }\end{array}$ & $\begin{array}{r}.78 \\
1.05\end{array}$ & $\begin{array}{l}1.01 \\
1.42\end{array}$ & $\begin{array}{l}1.37 \\
1.56\end{array}$ \\
\hline $\begin{array}{l}\text { Total } \\
\text { - hombres } \\
\text { - mujeres }\end{array}$ & $\begin{array}{l}.33 \\
.81\end{array}$ & $\begin{array}{r}.96 \\
1.66\end{array}$ & $\begin{array}{l}1.35 \\
2.13\end{array}$ \\
\hline
\end{tabular}

Tabla VII: Diferencias en el perfil EPQ-A y EBS entre los que nunca consumieron MDMA (abstinentes), los que probaron pero no continuaron (experimentadores), y los que habiendo probado continuaron consumiendo (reincidentes) (varones)

\begin{tabular}{|c|c|c|c|c|c|}
\hline & $\begin{array}{l}\text { Abstinentes } \\
(n=1375)\end{array}$ & $\begin{array}{l}\text { Experimentadores } \\
(n=38)\end{array}$ & $\begin{array}{l}\text { Reincidentes } \\
(n=35)\end{array}$ & $p^{*}$ & Grupos distintos \\
\hline EPQ-A & & & & & \\
\hline Neuroticismo & $12.58(4.93)$ & $12.80(5.67)$ & $12.57(5.62)$ & NS & No diferencias \\
\hline Intro-extroversión & $14.93(3.39)$ & $15.87(2.49)$ & $14.91(2.85)$ & NS & No diferencias \\
\hline Psicoticismo & $5.33(3.18)$ & $7.20(3.13)$ & $9.69(4.26)$ & .000 & 1 vs 2 vs 3 \\
\hline Búsqueda Sensaciones & & & & & \\
\hline BEM & $7.60(2.31)$ & $8.07(2.16)$ & $8.14(1.88)$ & NS & No diferencias \\
\hline BEX & $5.09(1.77)$ & $7.32(1.61)$ & $6.40(1.79)$ & .000 & 1 vs 2 vs 3 \\
\hline DES & $6.79(2.06)$ & $8.25(1.43)$ & $8.43(1.70)$ & .000 & 1 vs 2 y 3 \\
\hline SAP & $5.51(1.96)$ & $6.72(2.34)$ & $6.29(2.20)$ & .000 & 1 vs 2 y 3 \\
\hline Total & $24.98(5.35)$ & $30.15(4.75)$ & $29.29(5.67)$ & .000 & 1 vs 2 y 3 \\
\hline
\end{tabular}

*Anova Oneway (prueba post hoc: Duncan)

cerca del $4.0 \%$ de jóvenes entre 14-18 años referían haber consumido alguna vez MDMA (DGPNSD, 1995) y esa cifra ascendía a un $5 \%$ en 1996 (DGPNSD, 1997), para volver a descender a un $3.5 \%$ en 1998 (DGPNSD, 2000). En Austria, estudios a nivel provincial muestran prevalencias vida de $3.3 \%$ en jóvenes de 18-20 y esas cifras son más elevadas en la capital con una prevalencia vida del 6\% en jóvenes de 15-18 años (Haas, 1997). En el Reino Unido un estudio realizado durante 1996 sobre una amplia muestra de jóvenes de 15-16 años, pone de manifiesto que el 17\% de los chicos han usado alguna vez LSD, $14.5 \%$ anfetaminas y $9.2 \%$ éxtasis. Las prevalencias son inferiores en el caso de las chicas, $12.2 \%, 12.3 \%$ y $7.3 \%$, respectivamente (McC Miller y Plant, 1996).
Los estudios realizados en "poblaciones de riesgo" arrojan niveles más elevados de consumo de MDMA. Un estudio realizado en Amsterdam en 1995 entre frecuentadores de discotecas pone de manifiesto prevalencias vida del $52 \%$ y prevalencias durante el último año del $41 \%$ (Korf et al, 1996). Estudios realizados en el noreste italiano, en idéntico tipo de población, sugieren que entre el $9 \%$ y $65 \%$ de usuarios de discotecas refieren haber consumido la sustancia (Schifano et al, 1996).

A pesar de que autores como Gamella et al (1996) sugieren que la MDMA y sus análogos podrían estar convirtiéndose en una de las primeras drogas ilegales que empiezan a usar los jóvenes españoles, nuestros 
Tabla VIII: Diferencias en el perfil EPQ-A y EBS entre los que nunca consumieron MDMA (abstinentes), los que probaron pero no continuaron (experimentadores), y los que habiendo probado continuaron consumiendo (reincidentes) (mujeres)

\begin{tabular}{|l|l|l|l|l|l|}
\hline & $\begin{array}{l}\text { Abstinentes } \\
(n=1378)\end{array}$ & $\begin{array}{l}\text { Experimentadores } \\
(n=24)\end{array}$ & $\begin{array}{l}\text { Reincidentes } \\
(n=12)\end{array}$ & $p^{*}$ & Grupos distintos \\
\hline EPQ-A & $15.81(4.89)$ & $15.04(5.43)$ & $17.17(3.61)$ & NS & No diferencias \\
Neuroticismo & $14.65(3.52)$ & $15.40(3.03)$ & $16.67(2.19)$ & NS & No diferencias \\
Intro-extroversión & $3.80(2.56)$ & $5.52(3.50)$ & $5.33(3.55)$ & .001 & 1 vs 2 y 3 \\
Psicoticismo & & & & & \\
\hline Búsqueda Sensaciones & & $7.48(2.06)$ & $8.58(1.16)$ & NS & 1 vs 3 \\
BEM & $7.11(2.42)$ & $7.48(1.64)$ & $7.92(1.31)$ & .000 & 1 vs 2 y 3 \\
BEX & $5.72(1.71)$ & $7.56(1.45)$ & $7.67(1.50)$ & .000 & 1 vs 2 y 3 \\
DES & $5.73(2.23)$ & $6.00(1.53)$ & $6.25(2.83)$ & NS & No diferencias \\
SAP & $5.27(2.94)$ & $28.52(4.13)$ & $30.58(3.65)$ & .000 & 1 vs 2 y 3 \\
\hline Total & $23.77(5.41)$ & & & \\
\hline
\end{tabular}

*Anova Oneway (prueba post hoc: Duncan)

datos no confirman tales hallazgos, ya que la incorporación de nuestros jóvenes al consumo de MDMA es más tardía que en el caso de otras sustancias.

En consonancia con nuestros hallazgos, datos procedentes de múltiples países ponen de manifiesto la prácticamente inexistencia de consumidores "puros" de esta sustancia (Rakete y Flüsmeier, 1995; Bobes et al, 1996; Gamella et al, 1996; Korf et al, 1996; Meili, 1996; Schifano et al, 1996; Calafat et al, 1997; Tossman y Heckmann, 1997; DGPNSD, 1998), de modo que los usuarios de MDMA son generalmente policonsumidores de otras drogas, principalmente cannabis, anfetaminas, LSD, cocaína y alcohol.

Para la interpretación de los datos expuestos hay que tener presente que la prevalencia vida no refleja el patrón de consumo de una sustancia, de modo que dichas tasas pueden resultar alarmantes si se interpretan como patrones regulares de consumo. El uso crónico, repetido o actual de las drogas de síntesis es probablemente mucho más bajo y varía de acuerdo con el tipo de droga (Griffiths et al, 1997).

Antes de interpretar las diferencias psicológicas entre usuarios y no usuarios de MDMA, hemos de tener presentes ciertas características de las submuestras utilizadas en nuestro estudio. Uno de los grupos de no consumidores de MDMA (grupo 2) está constituido por personas que han consumido otras drogas, principalmente alcohol y cannabis. Por otra parte, el subgrupo de consumidores de MDMA (grupo 3) está formado por personas que han consumido otras sustancias - policonsumidores - que, además, muestran prevalencias de consumo de otras drogas claramente más elevadas que las halladas en el grupo 2.
Los datos aquí presentados ponen de manifiesto que los usuarios de MDMA obtienen puntuaciones más elevadas que los no consumidores de drogas ilegales y que el resto de policonsumidores no usuarios de MDMA, en una subescala del EPQ-A (psicoticismo). Esos datos concuerdan, al menos parcialmente, con los previamente reportados por Villa et al (1996) en consumidores habituales de MDMA.

Las puntuaciones obtenidas en todas las subescalas de la EBS (excepto la BEM en hombres y BEM y $S A B$ en mujeres) y en la puntuación total, son de igual modo más elevadas entre los usuarios de MDMA que en los otros dos grupos. Dichos resultados sugieren la existencia de diferencias entre los grupos en términos de búsqueda de sensaciones. Esas diferencias son lógicas, ya que, los grupos que refieren haber consumidos drogas ilegales (lo cual es un ejemplo de búsqueda de sensaciones) se muestran a sí mismos como más buscadores de sensaciones que los que nunca han consumido drogas ilegales. Además, si se tiene en cuenta que el número medio de sustancias ilegales consumidas alguna vez en la vida es más elevado entre los miembros del grupo 3 que los del grupo 2, es lógico que los primeros obtengan, en general, puntuaciones más altas en la EBS.

Numerosas investigaciones encuentran una relación consistente entre búsqueda de sensaciones y uso de drogas (Zuckerman et al, 1980, 1990; Bates et al, 1986; Luengo et al, 1996; Ravenna y Palmonari, 1996; Villa et al, 1996; López Miguel et al, 1997; Calafat et al, 1998). Zuckerman (1983), en una revisión de varios estudios, pone de manifiesto que la EBS (especialmente las subescalas DES y BEX) se relaciona con la frecuencia de consumos, siendo los psicoestimulantes los que muestran las mayores correlaciones. 
Teichman et al, (1989), consideran que la búsqueda de sensaciones sería mejor predictor del uso de drogas que la depresión o la ansiedad, y que permite identificar no consumidores, consumidores experimentales y todo tipo de usuarios de drogas. De igual modo, Jaffe y Archer (1987) consideran que la EBS es el mejor predictor de consumo en comparación con otros rasgos y escalas de personalidad. En este sentido, Luengo et al (1996), ponen de manifiesto que la búsqueda de sensaciones es un rasgo relevante a la hora de diferenciar a los adolescentes no-consumidores y a aquellos que incrementan el consumo a lo largo del segundo año del estudio: las puntuaciones medias en la EBS fueron claramente superiores en aquellos con patrones progresivos de consumo.

Calafat et al (1998), proponen dos posibles explicaciones a la relación existente entre búsqueda de sensaciones y uso de drogas. Zuckerman facilita la primera, al tratar de conectar las propiedades farmacológicas de cada sustancia con su teoría, y en una muestra compuesta por 80 usuarios de drogas encuentra que la búsqueda de sensaciones se relaciona positivamente con el uso de estimulantes y alucinógenos e inversamente con el uso de sustancias depresoras. Otra explicación, que quizás cuente con más apoyos, podría ser que el buscador de sensaciones sea un individuo interesado en los cambios que las drogas inducen en su comportamiento, considerando la clase de droga como un elemento secundario. En este sentido, la cultura y el mercado actual de drogas favorecen claramente el policonsumo y van en contra de una elección personalizada de drogas en función de la personalidad del usuario. Zuckerman (1983) también tiene en cuenta esa posibilidad cuando dice que las personas eligen una droga buscando un cambio en su estado afectivo más que unos efectos específicos.

Calafat et al (1998), encuentran que el subgrupo de consumidores ocasionales o experimentales de éxtasis ocupan una posición especial, siendo el único grupo de consumidores de éxtasis que muestra diferencias significativas con los no consumidores en todas las subescalas. El hecho de que las puntuaciones obtenidas por consumidores ocasionales o experimentales sean superiores a las encontradas en otros grupos de consumidores con frecuencias de consumos más elevadas, podría deberse a que la búsqueda de sensaciones sea más relevante en aquellos individuos que consumen drogas de modo ocasional, o de modo experimental, que en los que lo hacen de modo habitual o compulsivo, ya que al fin y al cabo éstos últimos buscan efectos que ya conocen, no son nuevas sensaciones para ellos y, por definición, un buscador de sensaciones, "siempre busca algo nuevo" (Zuckerman et al, 1972).

Aunque parece existir un acuerdo entre la mayoría de estudios en lo referente a búsqueda de sensaciones y uso de drogas, ese acuerdo desaparece cuando se trata de identificar que subescala de la EBS es la que más correlaciona con el consumo de sustancias.

En nuestro estudio, en líneas generales, las puntuaciones más elevadas en ambos grupos de consumidores de drogas ilegales se obtienen en las subescalas DES y BEM y las más bajas en la SAB. Los resultados obtenidos por los diferentes autores varían notablemente. Zuckerman et al (1972), en una muestra de varones, encuentran que el consumo de drogas correlaciona de modo significativo con todas las subescalas excepto con las DES, siendo las correla-

\section{Tabla IX: Diferencias, expresadas en desviaciones estándar, entre las puntuaciones obtenidas por los "abstinentes", "experimentadores" y "reincidentes" de las dos muestras (hombres y mujeres) y la población española de referencia en la EBS}

\begin{tabular}{|c|c|c|c|}
\hline & Abstinentes & Experimentadores & Reincidentes \\
\hline \multicolumn{4}{|c|}{ Búsqueda emociones (BEM) } \\
\hline - hombres & .31 & .49 & .52 \\
\hline - mujeres & .42 & .58 & 1.03 \\
\hline \multicolumn{4}{|c|}{ Búsqueda excitación (BEX) } \\
\hline - hombres & -.29 & .77 & .33 \\
\hline - mujeres & .17 & 1.09 & 1.33 \\
\hline \multicolumn{4}{|c|}{ Desinhibición (DES) } \\
\hline - hombres & .68 & 1.26 & 1.33 \\
\hline - mujeres & 1.44 & 2.40 & 2.46 \\
\hline \multicolumn{4}{|c|}{ Susceptibilidad aburrimiento (SAB) } \\
\hline - hombres & .87 & 1.42 & 1.22 \\
\hline - mujeres & 1.19 & 1.58 & 1.71 \\
\hline \multicolumn{4}{|l|}{ Total } \\
\hline - hombres & .57 & 1.38 & 1.25 \\
\hline - mujeres & 1.15 & 2.04 & 2.43 \\
\hline
\end{tabular}


ciones más elevadas con la BEX. Luengo et al (64) encuentran, en ambos sexos, que las subescalas DES y BEX son las más relacionadas con todos los tipos de consumos, mientras que la BEM es la que tiene las correlaciones más bajas. Ravenna y Palmonari (1997) encuentran, en ambos sexos, que BEM y BEX son las subescalas en las que se obtienen mayores puntuaciones. Un estudio previo realizado por nuestro grupo en consumidores habituales de MDMA (Villa et al, 1996) pone de manifiesto que las puntuaciones más elevadas se obtienen en la subescala BEX y las más bajas en la SAB. Finalmente, Calafat et al (1998), refieren que la escala más sensible a la hora de diferenciar consumidores de MDMA de no consumidores (en la que se encontraron las mayores diferencias entre los subgrupos) es la BEX, seguida de la SAB y la DES, siendo la BEM la subescala dotada de una menor sensibilidad.

\section{CONCLUSIONES}

La MDMA no es una de las drogas ilegales más utilizadas por los jóvenes estudiantes de secundaria, ocupando, además, una de las últimas posiciones en el ranking de edad de primer uso.

El consumo de MDMA es más prevalente dentro del sexo masculino.

El consumo de MDMA está fuertemente asociado con el consumo de otras drogas legales e ilegales.

Dicho policonsumo dificulta el establecimiento de un perfil psicopatológico propio de los consumidores de MDMA.

En este sentido, debemos ser cautos y sólo podemos afirmar que en nuestro estudio aquellos policonsumidores que además consumen MDMA, muestran un perfil diferencial. Dicho perfil está caracterizado por mayores niveles de búsqueda de sensaciones y psicotismo que en otros consumidores de drogas ilegales y que aquellos que no consumen drogas ilegales.

\section{BIBLIOGRAFIA}

Alvarez A, Gamella JF (1998): La experiencia del éxtasis. Un estudio antropológico de los efectos percibidos de la MDMA y sus análogos. En: Bobes J, Lorenzo P, Sáiz PA (eds.). Extasis (MDMA): Un abordaje comprehensivo. Barcelona: Masson, SA, pp.. 133-160.

Bates E, Labouvie EW, White HR (1986): The effect of sensation seeking needs on alcohol and marijuana use in adolescence. Bulletin of the Society of Psychologists in Addictive Behaviors; 5: 29-36.
Beck J: MDMA (1986): The popularisation and resultant implications of a recently controlled psychoactive substance. Contemporary Drug Problems; pp. 23-61.

Bobes J, González MP, Sáiz PA, Fernández-Miranda JJ, Pérez de los Cobos J (1995): Trastornos psiquiátricos relacionados con el uso de MDMA. En: Bobes J (ed.). Extasis. Aspectos farmacológicos, psiquiátricos y médico-legales. Barcelona: Ediciones en Neurociencias, pp. 47-76.

Bobes J, Sáiz PA, González MP, Bascarán MT, Pérez-Carral JR, López JL (1997): Valutazione sul consumo e abuso di droghe (Ecstasi) nei ragazzi che vengono incorporati nel servizio di leva. En: Proceedings Book of the International Confernce "Ecstasy e sostanze psichedeliche". Bolonia, 1996, pp. 29-59.

Bobes J, Sáiz PA, González MP, Bousoño M (1998a): Introducción: Aspectos históricos de la MDMA. En: Bobes J, Lorenzo P, Sáiz PA (eds.). Extasis (MDMA): Un abordaje comprehensivo. Barcelona: Masson, SA, pp. 1-13.

Bobes J, Sáiz PA, Bascarán MT, Fernández-Miranda JJ, López JL (1998b): Aproximación epidemiológica y tendencias de consumo de MDMA. En: Bobes J, Lorenzo P, Sáiz PA (eds.). Extasis (MDMA): Un abordaje comprehensivo. Barcelona: Masson, SA, pp. 107-131.

Bobes J, González MP, Sáiz PA, González JC (1998c): Psicopatología y trastornos psiquiátricos relacionados con el uso de MDMA. En: Bobes J, Lorenzo P, Sáiz PA (eds.). Extasis: Un abordaje comprehensivo. Barcelona: Masson, SA, pp. 173-197.

Calafat A, Sureda MP, Palmer A (1997): Características del consumo de éxtasis en una muestra de universitarios y usuarios de discoteca. Adicciones; 9 (4): 529-555.

Calafat A, Stocco P, Mendes F, Simon J, van de Wijngaart G, Sureda MP, Palmer A, Maalsté N, Zavatti P (1998): Characteristics and social representation of ecstasy in Europe. Valencia: IREFREA.

CIS (Centro de Investigaciones Sociológicas) (1994): Opiniones y comportamiento de los españoles ante el consumo de drogas, tabaco y alcohol. Estudio $n^{\circ}$ 2080. Madrid.

DGPNSD (Delegación del Gobierno para el Plan Nacional sobre Drogas) (1995): Encuesta sobre drogas en población escolar, 1994. Madrid: Ministerio de Justicia e Interior.

DGPNSD (Delegación del Gobierno para el Plan Nacional sobre Drogas) (1996a): Drogas de síntesis. Madrid: Ministerio de Justicia e Interior.

DGPNSD (Delegación del Gobierno para el Plan Nacional sobre Drogas) (1996b): Encuesta domiciliaria sobre uso de drogas. Madrid: Ministerio de Justicia e Interior.

DGPNSD (Delegación del Gobierno para el Plan Nacional sobre Drogas) (1997): Encuesta sobre drogas a la población escolar, 1996. Madrid: Ministerio de Interior.

DGPNSD (Delegación del Gobierno para el Plan Nacional sobre Drogas) (1998): Encuesta domiciliaria sobre 
consumo de drogas. Madrid: Ministerio de Justicia e Interior.

DGPNSD (Delegación del Gobierno para el Plan Nacional sobre Drogas) (2000): Encuesta sobre drogas en población escolar, 1998. Madrid: Observatorio Español. Plan Nacional sobre Drogas. Informe $n^{\circ} 3$.

Eysenck HJ, Eysenck SBG (1975): Eysenck personality questionnaire-junior (EPQ-J) \& adult (EPQ-A). London: Hodder and Stoughton Educational.

Gamella JF, Alvarez A, Meneses C (1996): Drogas de síntesis en España. Datos de una investigación antropológica. En: Libro de Abstracts de las XXIII Jornadas Nacionales de Socidrogalcohol. Oviedo: Socidrogalcohol, pp. 117-126.

Gamella JF, Alvarez A (1997): Drogas de síntesis en España. Patrones y tendencias de adquisición y consumo. Madrid: PNSD.

Giroud C, Augsburger M, Sadeghipour F, Varesio E, Veuthey JL, Rivier L (1997): Ecstasy - La situation en Suisse romande. Composition des saisies, analyse des échantillons biologiques et brève revue de son action pharmacologique et de sa toxicité. Schweiz Rundsch Med Prax; 86: 510-523.

Greer G, Tolbert R (1986): Subjective reports of the effects of MDMA in a clinical setting. Journal of Psychoactive Drugs; 18: 319-328.

Griffiths P, Vingoe L, Jansen K, Sherval J, Lewis R, Hartnoll $R$, Nilson M (1997): New trends in synthetic drugs in the European Union: Epidemiology and demand reduction responses. Lisbon: EMCDDA.

Grinspoon L, Bakalar JB (1986): Can drugs be used to enhance the psychotherapeutic process?. American Journal of Psychotherapy; XL: 393-404.

Grob Ch (1998): Investigación humana con MDMA. En: Bobes J, Lorenzo P, Sáiz PA (eds.). Extasis (MDMA): Un abordaje comprehensivo. Barcelona: Masson, SA, pp. 99-105.

Haas S (1997): Contry report A: Austria. En: Griffiths P, Vingoe $L$ (ed.). The use of amphetamines, ecstasy and LSD in the European Community: A review of data on consumption patterns and current epidemiological literature. Appendix I: Country reports. London: The National Addiction Centre, pp. 1-4.

Health Education Authority (1997): Drug use in England Results of the 1995 national drugs campaign survey. London: Health Education Authority.

Jaffe LT, Archer RP (1987): The prediction of drug use among college students from MMPI, MCMI and sensation seeking scales. Journal of Personality Assessment; 51: 243-253

Kontula O (1997): Contry report D: Finland. En: Griffiths P, Vingoe $L$ (ed.). The use of amphetamines, ecstasy and LSD in the European Community: A review of data on consumption patterns and current epidemiological literature. Appendix I: Country reports. London: The National Addiction Centre, pp. 18-29.

Korf DJ, Nabben T, Schreuders M (1996): Antenna 1995. Summary. Amsterdam: Jellinek Centrum.
López Miguel MJ, León Fuentes JL, Blanco Picabia A (1997): Búsqueda de sensaciones y consumo de drogas. Sevilla: Area de Personalidad, Evaluación y Tratamiento Psicológicos. Universidad de Sevilla. Tesis Doctoral.

Luengo A, Otero-López JM, Romero E, Gómez JA (1996): Efectos de la necesidad de búsqueda de sensaciones sobre la involucración en el consumo de drogas de los adolescentes. Análisis y Modificación de la Conducta; 22 (86): 683-708.

McC Miller P, Plant M (1996): Drinking, smoking, and illicit drug use among 15 and 16 year olds in the United Kingdom. British Medical Journal; 313: 394-397.

McCann U, Ricaurte G (1998): Aproximación a la neurobiología y neurotoxicidad comparada inducida por la MDMA. En: Bobes J, Lorenzo P, Sáiz PA (eds.). Extasis (MDMA): Un abordaje comprehensivo. Barcelona: Masson, SA, pp. 89-97.

McCann U, Szabo Z, Scheffel U, Dannals RF, Ricaurte GA (1998): Positron emission tomographic evidence of toxic effect of MDMA ("Ecstasy") on brain serotonin neurons in human beings. The Lancet; 352: 1433-1437.

Meili B (1996): National report of Switzerland. WHO Meeting on amphetamines, MDMA and other psychostimulants. Geneva, 12-15 november.

Morgan M, Grube JW (1994): Drinking among post-primary school pupils. Dublin: ESRI.

National Board of Health (1996): Annual Report on the state of the drugs problem in Denmark - National report for EMCDDA. Denmark, National Board of Health.

Olsson B (1997): Contry report N: Sweden. En: Griffiths P, Vingoe $L$ (ed.). The use of amphetamines, ecstasy and LSD in the European Community: A review of data on consumption patterns and current epidemiological literature. Appendix I: Country reports. London: The National Addiction Centre, pp. 131-138.

Pérez J, Torrubia R (1986): Fiabililidad y validez de la versión española de la Escala de Búsqueda de Sensaciones (Forma V). Revista Latinoamericana de Psicología; 18: 7-22.

Rabes M, Kraus L (1997): Contry report F: Germany. En: Griffiths $P$, Vingoe $L$ (ed.). The use of amphetamines, ecstasy and LSD in the European Community: $A$ review of data on consumption patterns and current epidemiological literature. Appendix I: Country reports. London: The National Addiction Centre, pp. 36-50.

Rakete G, Flüsmeier U (1995): Ecstasy-eine explorative studie zum konsum und midbrauch von ecstasy. Wiener Zeitschrift für Suchtforschung; 3:18.

Ravenna M, Palmonari A (1997): Rappresentazioni di sè e dello "sballo": studio su un campione di giovani studenti. En: Proceedings Book of the International Confernce "Ecstasy e sostanze psichedeliche". Bolonia, 1996.

Ricaurte GA, Finnegan KT, Irwin I, Langston JW (1990): Aminergic metabolites in cerebrospinal fluid of humans 
previously exposed to MDMA: Preliminary observations. Ann NY Ac Sci; 600: 699-710.

Sáiz PA, González MP, Bousoño M, Bobes J (1998): Magnitud e impacto de las drogas de síntesis. Coloquios en Drogodependencias; 5: 4-7.

Sandwijk JP, Cohen PDA, Musterd S (1991): Licit and illicit drug use in Amsterdam. Report of a household survey in 1990 on the prevalence of drug use among the population of $\mathbf{1 2}$ years and over. Amsterdam: Instituut voor Sociale Geografie, Universiteit van Amsterdam.

Sandwijk JP, Cohen PDA, Musterd S (1995): Licit and illicit drug use in Amsterdam II. Report of a household survey in 1994 on the prevalence of drug use among the population of $\mathbf{1 2}$ years over. Amsterdam: Instituut voor Sociale Geografie, Universiteit van Amsterdam.

Schifano F, Di Furia L, Miconi L, Bricolo R (1996): MDMA (ecstasy) ed altri amfetamino-simili: aspetti epidemiologici e clinici. En: Proceedings Book of the 2nd Congresso Nazionale di Medicina Nelle Dipendence. Verona: Gruppo Intersert Collaborazione Scientifica (GICS), pp. 97-108.

SFA / ISPA (1996): Ecstasy in der Schweiz, die erste repräsentative Studie zur Modedroge. Lausanne: SFA (Schweizerische Fachstelle für Alkohol - und andere Drogenprobleme).

Shulgin A, Nichols DE (1978): Characterization of three new psychotomimetics. En: Stillman R, Willete R (eds.). The psychopharmacology of hallucinogens. New York: Pergamon Press, pp. 74-83.

Smart RG, Hughes PH, Johnston LD, Anumonye A, Khant U, Medina ME, Navaratnam V, Poshyachinda V, Varma VK,
Wadud KA (1980): Méthodologie pour des enquètes sur I'usage des drogues chez les étudiants. Publication offset $N^{\circ} 50$. Genève: OMS.

Teichman M, Barnea Z, Rahav G (1989): Sensation Seeking, state and trait anxiety, and depressive mood in adolescent substance users. Int J Addict; 24 (2): 87-99.

Tossman P, Heckmann W (1997): Drug affinity in the techno party scene - preliminary results. Berlin: SPI Research.

Villa A, Sáiz PA, González MP, Fernández JJ, Bousoño M, Bobes J (1996): Hacia una definición del perfil psicosocial y psicopatológico de los consumidores de éxtasis. Anales de Psiquiatría; 12: 183-189.

Zuckerman M (1983): A biological theory of sensation-seeking. En: Zuckerman M (ed.). Biological bases of sensation-seeking, impulsivity, and anxiety. Hillsdale: Erlbaum, pp. 37-76.

Zuckerman M, Bone R, Neary R, Mangelsdorff D, Brustman B (1972): What is the sensation seeker? Personality trait and experience correlates of the sensation-seeking scales. J Consult Clin Psychol; 39: 308-321.

Zuckerman M, Eysenck SBG, Eysenck HJ: Sensation-seeking in England and America: Cross-cultural, age and sex comparisons. J Consulting Clinical Psychology 1978; 46: 139-149.

Zuckerman M, Neeb N (1980): Demographic influences in sensation-seeking and expression of sensation seeking in religion, smoking and driving habits. Personality and Individual Differences; 1: 197-206.

Zuckerman M, Ball S, Black J (1990): Influences of sensation seeking, gender, risk appraisal and situational motivation on smoking. Addictive Behaviors; 15: 209-220. 
